\title{
Relación del equilibrio ácido-base y supervivencia en adultos con diagnóstico de trauma en la unidad de cuidados intensivos del Hospital Universitario San Jorge. 2009-20 10, Pereira, Colombia
}

\author{
Raúl Eduardo Aristizabal ${ }^{1}$ \\ José William Martínez ${ }^{2}$ \\ Mauricio Montoya-Cañón ${ }^{3}$ \\ Luis Alfonso Valencia ${ }^{4}$ \\ Luis Felipe Calvo-Torres ${ }^{5}$ \\ Oscar Barbosa-Gantiva ${ }^{6}$ \\ Vanesa Hincapié
}

\footnotetext{
1. Médico. Intensivista. Unidad de Cuidados Intensivos. Clínica San Rafael. Pereira. Risaralda. Colombia.

2. Médico. Doctor en Epidemiología. Programa de Medicina y Cirugía. Facultad de Ciencias de la Salud. Universidad Tecnológica de Pereira. Pereira. Risaralda. Colombia.

3. Médico. Grupo de Investigación en Farmacoepidemiología y Farmacovigilancia. Universidad Tecnológica de Pereira. Hospital Local San Juan de Dios. Pensilvania. Caldas. Colombia.

4. Médico. Universidad Tecnológica de Pereira. Pereira. Colombia.

5. Médico. Grupo de Investigación en Farmacoepidemiología y Farmacovigilancia. Universidad Tecnológica de Pereira. Hospital Nazareth. Quinchía. Risaralda. Colombia.

${ }^{6}$ Médico. Universidad Tecnológica de Pereira. Pereira. Risaralda. Colombia.

${ }^{7}$ Estudiante de XIII nivel de medicina. Facultad Ciencias de la Salud. Universidad Tecnológica de Pereira. Pereira. Colombia.
}

Correspondencia: Dr. Mauricio Montoya. Dirección: Calle 21 \# 24-230, Edificio Los Álamos. Pereira. Colombia.

Correo electrónico: mmcmarzo@gmail.com

\section{RESUMEN}

Introducción: El trauma es un problema de salud pública en Colombia, siendo la primera causa de mortalidad en jóvenes con una tasa de incidencia de 331,99 casos por 100000 habitantes, de los cuales aproximadamente el 30\% mueren en la unidad de cuidados intensivos. Estos pacientes de acuerdo a su severidad requerirán estabilización de signos vitales y monitoreo estrecho que incluye el estado ácidobase. Objetivo: Establecer la relación entre el desenlace y las variables del estado ácido base de los pacientes con diagnóstico de trauma de la unidad de cuidados intensivos del Hospital Universitario San Jorge de Pereira, Colombia. Materiales y métodos: Se realizó un estudio tipo cohorte prospectivo en la unidad de cuidados intensivos, en pacientes con diagnóstico de trauma a los que se les tomó muestra de sangre arterial para gases sanguíneos al momento de su ingreso y se monitorearon a través del tiempo hasta el egreso. Resultados: Se incluyeron 38 pacientes con trauma donde el 68,4\% fueron hombres. El promedio de edad fue de $35 \pm 19$ años y el 18,4\% de los pacientes que ingresaron fallecieron. Se realizó un análisis univariado, el cual mostró relación individual entre el desenlace y las variables sodio, saturación arterial de oxígeno, presión alveolar de oxígeno y el puntaje de APACHE II; en el modelo de regresión de Cox se encontró relación con las variables presión alveolar de oxígeno, presión arterial de oxígeno, frecuencia cardiaca y relación arterio-alveolar de oxígeno. Conclusión: Se encontró que las variables presión alveolar de oxígeno, presión arterial de oxígeno, frecuencia cardiaca y relación arterio-alveolar de oxígeno del análisis de los gases arteriales, tienen correlación con la supervivencia de los pacientes en estado crítico. Se hace una invitación para utilizar este examen paraclínico. MÉD UIS. 2015;28(3):273-80.

Palabras clave: Heridas y traumatismos. Cuidados intensivos. Equilibrio Ácido-Base. Oxigenación. 


\title{
Relation of acid-base equilibrium and survival in adults with diagnosis of trauma in intensive care unit in San Jorge University Hospital. 2009-2010, Pereira, Colombia
}

\begin{abstract}
Introduction: Trauma is a public health problem in Colombia and is the first cause of death in young people with an incidence rate of 331.99 cases per 100000 inhabitants, of which approximately $30 \%$ die in the intensive care unit. These patients according to their severity will require stabilization of vital signs and close monitoring which includes the acid-base status. Objetive: To establish the relationship between outcome and variables of acid-base equilibrium of those patients diagnosed with trauma in the intensive care unit of the Hospital Universitario San Jorge in Pereira, Colombia. Materials and Methods: A prospective cohort study in type was performed on the intensive care unit with general trauma patients who were taking blood gases on admission to the unit and were monitored over time until discharge. Results: The population of the study were 38 patients with trauma, $68.4 \%$ were men. Average age was $35 \pm 19$ years and $18.4 \%$ of patients admitted died. The univariate analysis was found individual relationship with outcome and variables such as sodium, arterial oxygen saturation, alveolar oxygen pressure and APACHE II score. In the Cox regression model it was found a relationship between outcome and variables such as alveolar oxygen pressure, pressure oxygen pressure, heart rate and arterial-alveolar ratio of oxygen. Conclusion: Variables found that alveolar oxygen pressure, arterial oxygen pressure, heart rate and arterial-alveolar oxygen ratio of arterial blood gas analysis, correlate with survival of patients in critical condition. An invitation to use this paraclinical test is done. MÉD UIS. 2015;28(3):273-80.
\end{abstract}

Keywords: Wounds and injuries. Intensive Care. Acid-Base Equilibrium. Oxygenation.

¿Cómo citar este artículo?: Aristizabal RE, Martínez JW, Montoya-Cañón M, Valencia LA, CalvoTorres LF, Barbosa-Gantiva O, et al. Relación del equilibrio ácido-base y supervivencia en adultos con diagnóstico de trauma en la unidad de cuidados intensivos del Hospital Universitario San Jorge. 2009-2010, Pereira, Colombia. MÉD UIS. 2015;28(3):273-80.

\section{INTRODUCCIÓN}

En Colombia el trauma es considerado un problema de salud pública, corresponidendo a la primera causa de muerte en la población joven específicamente en las tres primeras décadas de vida y la tercera causa de muerte en población general independiente de la edad'. El substrato del trauma en Colombia está dado por la violencia interpersonal-homicidio, violencia intrafamiliar, sexual, los accidentes de tránsito, el suicidio y las lesiones accidentales. Las estadísticas de Colombia muestran una mayor prevalencia en el sexo masculino según la causa, exceptuando la violencia intrafamiliar y sexual ${ }^{2}$.

La incidencia de violencia interpersonal en Colombia para el año 2011 fue de 331,99 casos por 100000 habitantes, con un aumento del $5 \%$ con respecto al año 2010; en el caso de las lesiones no fatales se registraron 88,6 casos por 100000 habitantes. El homicidio registró una tasa de mortalidad de 35,95 casos por 100000 habitantes, mientras que los accidentes de tránsito registraron una tasa de mortalidad de 12,6 por 100000 habitantes; finalmente se presentó una tasa de 4 suicidios por 100000 habitantes².

En el departamento de Risaralda se encontró en el año 2010 un porcentaje de lesiones interpersonales del 36,7\% y para las lesiones en accidentes de tránsito un $25,7 \%$, siendo estas las causas de mayor frecuencia para lesiones no fatales. En cuanto a las lesiones fatales se registró una disminución, con respecto al año anterior, con un porcentaje de $10,3 \%$, siendo la principal causa el homicidio en el $62,0 \%$ de los casos. En Pereira la tasa de muertes violentas en el 2011 fue del 72,01 por 100000 habitantes, pero cabe destacar que al menos siete ciudades del departamento registraron una incidencia mayor, con respecto a esta capital².

En el año 2013 se presentaron 1553 casos en total de violencia interpersonal, 986 casos sexo masculino y 567 casos en el sexo femenino, con una tasa total de 334,18 por 100000 habitantes en Colombia. Bogotá ocupa el primer lugar con 45433 casos, Cali ocupa el segundo lugar con 6933 casos, Medellín el tercero con 6490 casos, seguido de Barranquilla con $3775 \operatorname{casos}^{3}$. Para el 2015, Risaralda se situó entre los diez primeros departamentos con mayor tasa de homicidio, ocupando el octavo lugar a nivel de Colombia. Para este mismo año se observó en Pereira una tasa de homicidios del 39,38 casos por 100000 habitantes en total, teniendo mayor representación en los hombres con una tasa del 77,38 por 100000 habitantes, respecto a las mujeres que fue de 4,92 por 100000 habitantes 3 . 
El 28,5\% de las muertes por accidentes de tránsito se concentranendiez ciudadesenordendemayoramenor incidencia, Bogotá D.C, Cali, Medellín, Villavicencio, Cartagena, Cúcuta, Pereira, Bucaramanga, Ibagué y Valledupar. Para el caso específico de Pereira la tasa de mortalidad por accidentes de tránsito es de 18,29 por 100000 habitantes, (75 casos en hombres, tasa de 33,94 por 100000 habitantes y en mujeres 10 casos con tasa de 4,10 por 100000 habitantes) ${ }^{3}$. El $50,06 \%$ de lesionados se concentran en 15 ciudades, encontrandose la mayor tasa de incidencia en Bogotá D.C, seguido de Medellín, Cali, Bucaramanga, Ibagué, Pereira, Barranquilla, Manizales, Armenia, Neiva, Villavicencio, Cartagena, Itagüí, Madrid (Cundinamarca) y Cúcuta. Pereira ocupa el sexto lugar con una tasa de 259,51 por 100000 habitantes (746 casos en hombres, tasa 337,57 por 100000 habitantes, 460 casos en sexo femenino con tasa de 188,74 por 100000 habitantes) ${ }^{3}$.

Se registraron para el año 2014 muertes por lesiones accidentales con una tasa de 7,32 por 100000 habitantes y tasa de lesionados de 12,27 por 100000 habitantes, presentandose mayor distribución en el sexo masculino para las dos categorías. Adicionalmente, las ciudades capitales con las cinco tasas más altas para muertes accidentales fueron Mocoa (24,64), Quibdó (20,82), Puerto Carreño $(13,11)$, Yopal $(12,01)$ y Arauca $(11,63)$, y para las lesiones no fatales fueron Bogotá, D.C $(119,18)$, San José del Guaviare $(43,24)$, Arauca $(33,72)$, Armenia $(25,89)$ y Neiva $(22,20)$; correspondiendo con el promedio nacional de 27,363. Adicionalmente, en Pereira se presentaron 5,16 casos por 100000 habitantes de suicidio, con 24 casos en total (19 hombres y 5 mujeres), en contraste con ciudades principales del país que presentaron un mayor número de casos, Bogotá (236), Medellín (124), Cali (64), Barranquilla (56) e Ibagué con 42 casos $^{3,4}$.

Las consecuencias de este fenómeno se ven reflejadas en el ámbito económico por la disminución en la productividad personal y de la comunidad, no solo por los años de vida potenciales perdidos, sino también por los años de vida ajustados por incapacidad; y este último, a su vez con repercusiones como la disminución de la calidad de vida que afecta la familia, el paciente y la sociedad'. Dada la importancia del trauma en el contexto actual y que muchos de estos pacientes de acuerdo a su severidad van a necesitar un manejo que garantice la estabilidad de sus signos vitales, que evite llegar al shock y finalmente a la muerte ${ }^{5}$, se hace necesario un manejo completo por parte del equipo de cuidado crítico, en el cual el monitoreo del estado ácido-base juega un papel fundamental para evaluar la deuda de oxígeno y los procesos que de esta se derivan ${ }^{6}$. Se realizó esta investigación con el objetivo de evaluar la relación del equilibrio ácido-base y la supervivencia de pacientes adultos que ingresan a la Unidad de Cuidados Intensivos (UCI) con diagnóstico de trauma.

\section{Materiales y Métodos}

\section{ESTUDIO Y POBLACIÓN}

Se realizó un estudio tipo cohorte, prospectivo, concurrente con una fecha de corte puntual correspondiente a la fecha y hora de ingreso del paciente a la UCI entre el primero de octubre de 2009 y 30 de marzo de 2010. El desenlace correspondió al estado vital al egreso de la $\mathrm{UCl}$, la exposición fue la intervención diagnóstica (determinación de gases arteriales) de los pacientes que ingresaron a la $\mathrm{UCI}$ y el tiempo de observación fue la diferencia entre la fecha de egreso y la fecha de ingreso a la UCl. Los criterios de inclusión en el estudio fueron pacientes que ingresaron a la $\mathrm{UCl}$ por diagnóstico de trauma por cualquier causa (de acuerdo al CIE 10) y tener una o más muestras de gases arteriales recolectada, no se excluyó ningún paciente en el estudio. La población correspondió a los pacientes que ingresaron a la UCI del Hospital Universitario San Jorge de la ciudad de Pereira, centro de referencia del Eje Cafetero colombiano, a quienes se les tomaron muestras de gases arteriales y venosos.

\section{ANÁLISIS DE VARIABLES}

La información se obtuvo de la historia clínica digital del programa Dinámica Gerencial®. Los valores de gases sanguíneos fueron tomados por la máquina de gases GEM PREMIER 3000®7, la cual necesita de $150 \mu \mathrm{L}$ de sangre total (arterial, venosa o capilar). En algunos casos la muestra puede ser tomada de una línea venosa central, para medir las diferentes variables. Para que la variabilidad fuera atribuida a los pacientes y no a los encuestadores, se realizó un instructivo en el cual se determinó que los datos debían recolectarse todos los días, en parejas, por un grupo de estudiantes de medicina. Todo el trabajo se realizó bajo la supervisión del médico intensivista encargado de la investigación.

Los datos fueron recolectados en una base de datos del programa Excel en la que se introdujo información de los gases sanguíneos tales como: Fracción 
Inspirada de $\mathrm{O}_{2}$ ( $\left.\mathrm{FiO}_{2}\right), \mathrm{pH}$, Presión Parcial de $\mathrm{CO}_{2}$ ( $\left.\mathrm{PCO}_{2}\right)$, presión parcial de $\mathrm{O}_{2}$, valores sericos de $\mathrm{Na}+, \mathrm{K}+, \mathrm{Ca}+2$, concentración total de hemoglobina, Base Exceso (BE) del fluido extracelular, BE de concentración en sangre, saturación de O2, bicarbonato, bicarbonato estándar, Presión Alveolar de $\mathrm{O}_{2}$ ( $\mathrm{PAO} 2$ ), e índice arterio/alveolar de $\mathrm{O}_{2}$ ( $\mathrm{PaO} 2 /$ $\mathrm{PAO} 2)$. La historia clínica correspondió a una fuente adicional de información, de la cual se extrajeron las variables sociodemográficas del estudio tales como: edad, sexo y otras como fecha de ingreso al hospital, fecha de ingreso a la $\mathrm{UCl}$, fecha de egreso de la UCI, las comorbilidades y el estado del paciente al egreso. La consulta de la historia clínica se realizó para verificar los datos tomados.

Durante el tercer trimestre del año 2012, se recogieron datos de las historias clínicas de los mismos pacientes con el fin de estandarizarlos por medio de la escala APACHE II, índice de shock y el Revised Trauma Score (RTS). Esta información se recogió con el fin de hacer comparación del riesgo de mortalidad de los pacientes, puesto que no se recolectaron inicialmente; además se consideró que la literatura mundial hace un análisis basado en escalas de riesgo, por lo cual se retomaron los datos necesarios para aplicarlos en este artículo ${ }^{21,22}$.

\section{ANÁLISIS ESTADÍSTICO}

Para el análisis estadístico se utilizó el programa SPSS $20^{\circledR}$. Se evaluó la asociación entre las variables demográficas, las arrojadas por la tirilla de gases arteriales y las escalas APACHE II, índice de shock y RTS, cada uno de los componentes de dichas escalas fue analizado usando modelos como ANOVA y se consideró significativa la asociación con valores de $p<0,05$. Luego se realizó un análisis utilizando el modelo Kaplan-Meier y finalmente se llevo a cabo un análisis multivariado, aplicando el modelo de regresión de Cox para el control de confusores y predictivas de mortalidad.

Para el médico es de gran importancia conocer el estado del equilibrio ácido-base de los pacientes y para esto requiere de la relación entre algunas variables de los gases sanguíneos, tales como el $\mathrm{pH}$, $\mathrm{PCO}_{2}$, el $\mathrm{HCO}_{3}$ y la $\mathrm{BE}$. Estos valores se relacionan para configurar el diagnóstico del trastorno del equilibrio ácido-base de los pacientes en un momento determinado, información importante para la toma de decisiones diagnósticas y orientar las opciones terapéuticas, especialmente en la UCI. En este estudio el diagnóstico del equilibrio ácido-base se hizo de acuerdo a la información de la Figura $1^{8-10}$.

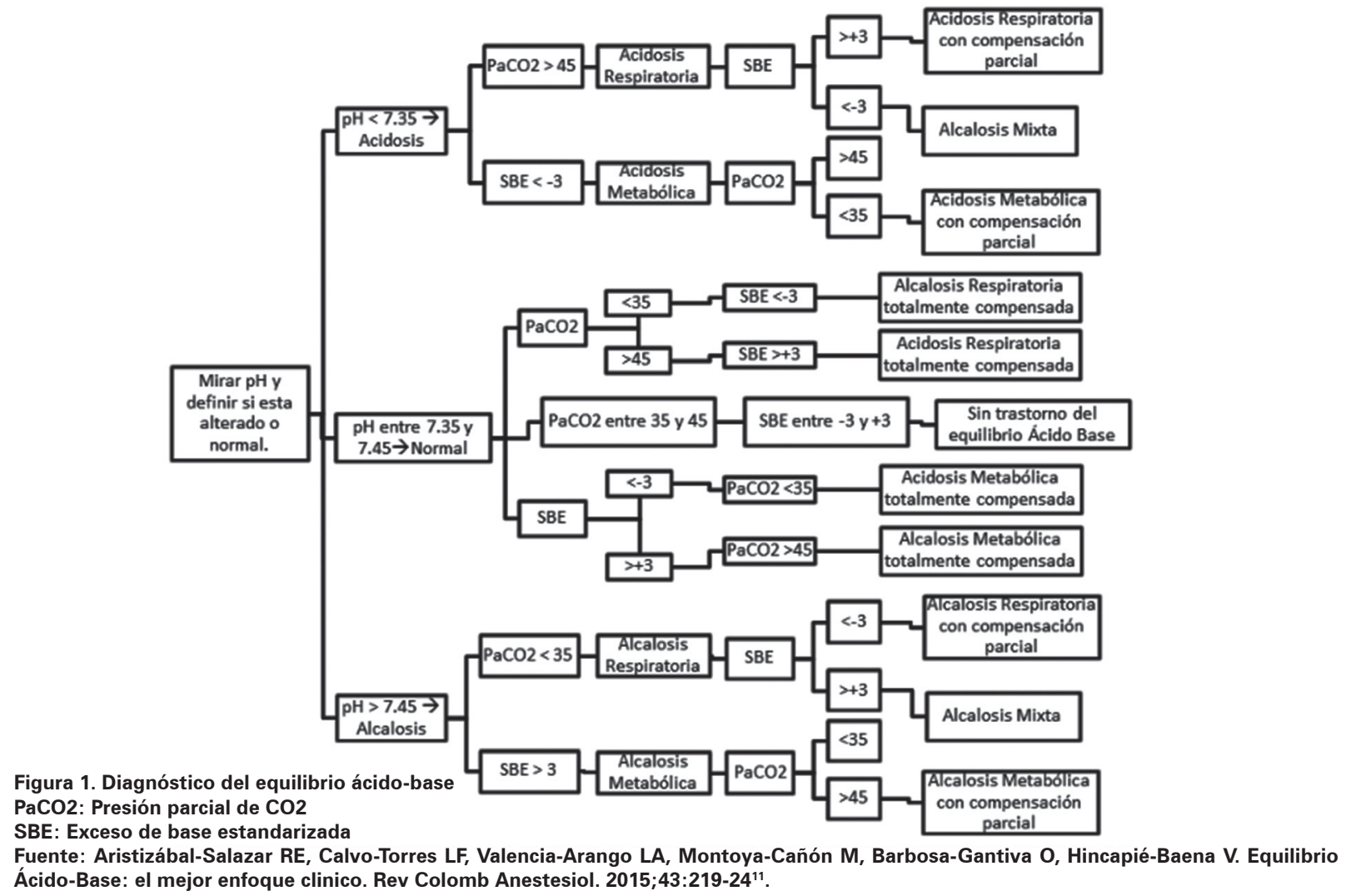




\section{SEPTIEMBRE-DICIEMBRE}

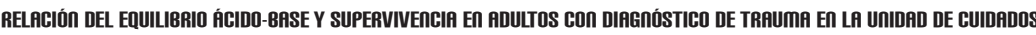
IOTENSIVOS DEL HOSPITAL UNIVERSITARIO SAI JORGE. २OO१-2010, PEREIRA, COLOMBIA

Existen otras variables de interés para los médicos intensivistas, como BE que es definida como la cantidad de ácido o de base necesaria para conseguir que un litro de sangre con una determinada concentración de hemoglobina y a $37{ }^{\circ} \mathrm{C}$ tenga un $\mathrm{pH}$ de $7,40^{12,13}$. Esta variable se divide en $\mathrm{BE}$ de concentración en sangre y BE del fluido extracelular ${ }^{14}$ cuya diferencia radica en el cálculo realizado por la máquina, teniendo en cuenta la concentración total de hemoglobina para BE de concentración en sangre, y en BE del fluido extracelular se toma una concentración de hemoglobina de $5 \mathrm{~g} / \mathrm{dL}$ que refleja la concentración total de hemoglobina en el volumen que se distribuye el bicarbonato en todo el líquido extracelular ${ }^{15,16}$.

Además, el cortocircuito intrapulmonar cuantifica el grado de desequilibrio entre la ventilación y la perfusión pulmonar, el $\mathrm{PaO}_{2} / \mathrm{PAO}_{2}$ representa el índice arterio/alveolar de oxígeno cuyo valor normal es de 0,8 y el $\mathrm{PaO}_{2} / \mathrm{FiO}_{2}$ que simboliza el índice de oxigenación que es muy utilizado por su correlación (hasta del $80 \%$ ) con el cortocircuito intrapulmonar ${ }^{17,18}$. Con el fin de estandarizar los análisis estadísticos en una población heterogénea como lo es la población con diagnóstico de shock, debido a sus múltiples etiologías desde todo punto de vista (edad, patología, sexo entre otras) como lo es la de la $\mathrm{UCl}$, fue necesario utilizar índices de severidad de enfermedad para ajustar esta a otras variables en el impacto que tienen sobre la mortalidad. La escala Acute Physiology And Chronic Health Evaluation II (APACHE II), incluye una medida numérica de la severidad del trastorno fisiológico; pero además incluye el grado y peso de otras variables como la edad, las enfermedades prexistentes y la escala de coma de Glasgow, por lo que ha demostrado ser superior y mejor índice para comparar probabilidad de mortalidad en diferentes $\mathrm{UCl}$ independiente del tipo de morbilidad ${ }^{19,20}$.

\section{Resultados}

La UCl del Hospital Universitario San Jorge es una unidad general que cuenta con 12 camas. Durante el período del estudio hubo un total de 2160 camas disponibles, con un promedio de diez pacientes día en la unidad, con un porcentaje de ocupación del $88,5 \%$ y el promedio de días estancia fue de 9,7. De los 211 pacientes que recibió la $U C l$ del Hospital Universitario San Jorge durante el periodo de estudio, 38 ingresaron con un diagnóstico de trauma. De estos 38 pacientes con trauma, El 68,4\% (26) fueron hombres. El promedio de edad fue de $35 \pm 19$ años y solo el $18,4 \%$ (7) de los pacientes que ingresaron fallecieron (Ver Tabla 1). Las variables que presentaron mayor significancia estadística en el análisis univariado ajustadas al desenlace (fallecer), las cuales fueron tomadas a partir de la tirilla de gases arteriales y de la historia clínica de ingreso a la $\mathrm{UCl}$, fueron $\mathrm{Na}^{+}(p=0,015), \mathrm{SO}_{2}(p=0,011), \mathrm{PAO}_{2}(p=0,031)$ y la escala de APACHE II $(p=0,031)$.

Tabla 1. Caracterización de la población del estudio

$$
\text { Sexo }
$$

\begin{tabular}{|c|c|c|c|c|c|}
\hline \multicolumn{2}{|c|}{} & Frequency & Percent & $\begin{array}{c}\text { Valid } \\
\text { Percent }\end{array}$ & $\begin{array}{c}\text { Cumulative } \\
\text { Percent }\end{array}$ \\
\hline \multirow{3}{*}{ Valid } & F & 12 & 31.6 & 31.6 & 31.6 \\
\cline { 2 - 6 } & $M$ & 26 & 68.4 & 68.4 & 100.0 \\
\cline { 2 - 6 } & Total & 38 & 100.0 & 100.0 & \\
\hline
\end{tabular}

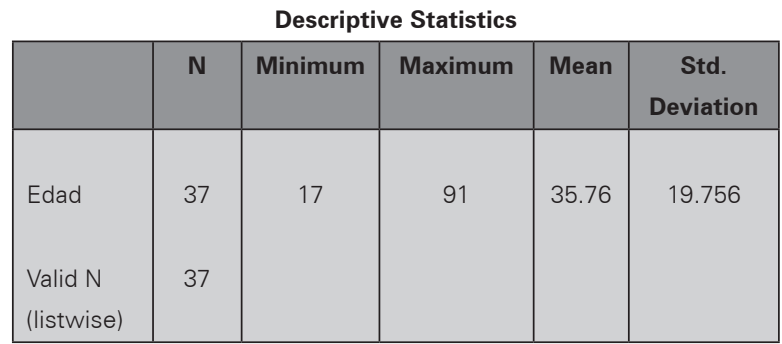

Fuente: Autores.

Se analizó la supervivencia mediante el modelo de Kaplan-Meier, el cual consiste en un estimador no paramétrico que permite establecer la probabilidad independiente de cada sujeto de sufrir un suceso en un periodo de tiempo, en este caso morir en un periodo de días; tiene gran importancia puesto que brinda mayor grado de certeza en poblaciones pequeñas al no agrupar en intervalos los tiempos de sobrevida. Se encontró que a pesar de la censura, la mayor probabilidad de morir se encuentra, para hombres y mujeres, al cuarto y séptimo día de ingreso a $\mathrm{UCl}$ respectivamente, no se halló relación estadísticamente significativa entre la supervivencia del paciente que ingresa con diagnóstico de trauma a la $\mathrm{UCl}$ y los diagnósticos de acidosis respiratoria Hazard Ratio (HR) de 5,0 ( $p=$ no se pudo establecer por censura en la muestra); alcalosis respiratoria HR de 3,5 $(p=0,85)$; acidosis metabólica HR de 2,16 $(p=0,87)$; alcalosis metabólica HR de 4,6 $(p=0,77)$ y demás diagnósticos mixtos (Ver Figura 2). 


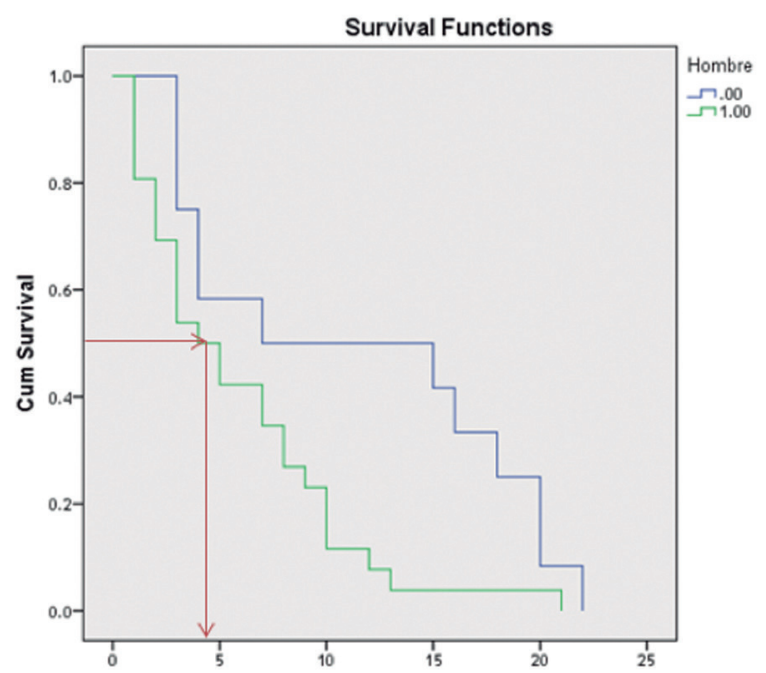

Figura 2. Modelo Kaplan-Meier Línea azul: Mujeres/ Línea verde: Hombres Fuente: Autores.

En cuanto al análisis multivariado efectuado con el modelo de regresión lineal de Cox (Ver Tabla 2), las variables $\mathrm{PAO}_{2}$ y $\mathrm{PaO} 2 / \mathrm{PAO}_{2}$ se asociaron con una mayor probabilidad de sobrevida y por el contrario, $\mathrm{PaO} 2$ y Frecuencia Cardiaca (FC) tienen una asociación negativa para la sobrevida, todo esto en un modelo biológicamente explicativo pero estadísticamente inestable debido a intervalos de confianza (IC 27,5 $65028,1)$ y a valores $\beta(7,19)$ amplios observados en la relación $\mathrm{PaO}_{2} / \mathrm{PAO}_{2}$, en consecuencia a un valor sobrestimado dado por sus componentes, los cuales se encuentran incluidos en el modelo como variables independientes.

\section{Discusión}

Los datos de mortalidad mencionados en las series, están relacionados con el fallecimiento de los pacientes en otras áreas tales como la sala de trauma, el quirófano e incluso en la atención prehospitalaria, siendo más reducido el número de pacientes que ingresan a la $\mathrm{UCl}$ con diferentes diagnósticos, pero bajo el denominador común de trauma, que hace de esta una población variada ${ }^{23}$.

Al comparar los resultados obtenidos en este estudio con otros estudios similares, se evidencia la importancia del oxígeno en todos los pacientes críticamente enfermos, con o sin trauma, ya que todos comparten como variables asociadas a mortalidad las relacionadas con el aporte de oxígeno a los tejidos, como lo son la PAO2, PaO2/PAO2 entre otras. La FC en un paciente críticamente enfermo con diagnóstico de trauma de cualquier causa, está asociada con desenlace de mortalidad. Tanto en $\mathrm{UCl}$ como en salas de trauma y en la escena pre hospitalaria, todas estas variables se convierten en blancos terapéuticos claves para mejorar el pronóstico de los pacientes con trauma críticamente enfermos ${ }^{24}$.

De acuerdo con la evidencia existente cabe resaltar la importancia que tienen las escalas de Trauma y APACHE II, los cuales se comportan como buenos predictores de mortalidad en pacientes con trauma en estado $\mathrm{Crítico}^{25}$. En el presente estudio, la FC alta se obseva como variable con significancia estadística para un resultado final desfavorable, como se evidenció en el modelo de regresión logística de Cox. Lo anterior es muy importante en el contexto del paciente en cuidado crítico, pues una vez resuelta la mayor parte de las condiciones quirúrgicas, es admitido a la $\mathrm{UCl}$ para continuar la reanimación guiada por objetivos; pero luego de haber controlado otras causas de taquicardia (dolor, fiebre, etc.), si esta persiste, es sensible para indicar que algo en el paciente anda mal y que probablemente, no todas las condiciones potencialmente mortales fueron resueltas y requiere de nuevas intervenciones.

Tabla 2. Variables asociadas a mortalidad en un modelo de regresión lineal Cox, Pereira 2010

\begin{tabular}{|l|c|c|c|c|c|c|}
\hline \multirow{2}{*}{ Variable } & \multirow{2}{*}{ B } & \multirow{2}{*}{ Sig } & OR & \multicolumn{2}{c|}{ IC del 95\% } & Variable \\
\cline { 5 - 7 } & & & & Inferior & Superior & Predictora \\
\hline $\mathrm{PaO} 2 \mathrm{C}$ & $-0,014$ & 0,014 & 0,986 & 0,975 & 0,997 & Mortalidad \\
\hline $\mathrm{PAO} 2$ & 0,009 & 0,008 & 1,009 & 1,002 & 0,015 & Sobrevida \\
\hline $\mathrm{PaO} 2 / \mathrm{PAO} 2$ & 7,199 & 0,000 & $1,338,158$ & 27,537 & $65,028,158$ & Sobrevida \\
\hline FC & $-0,022$ & 0,020 & 0,978 & 0,960 & 0,997 & Mortalidad \\
\hline
\end{tabular}

B: coeficiente de regresión; Sig: nivel de significancia; OR: odds ratio, en inglés; IC del $95 \%$ : intervalo de confianza del $95 \%$ Fuente: Autores. 
Esta referenciado el hecho de que ser un paciente masculino con afectación por trauma es un factor de riesgo'. La probabilidad de morir en función del tiempo es similar tanto para hombres como mujeres en los primeros cuatro días, no obstante a partir del quinto día se observa que las mujeres entran en una especie de fase de meseta para la supervivencia hasta el séptimo día, mientras que en los hombres la mortalidad sigue un patrón similar al de los primeros cinco días.

Al observar una variable con significancia, como la $\mathrm{PAO} 2$, permite inferir aspectos de disponibilidad de oxígeno a nivel alveolar y si posee buena ventilación; se puede identificar como un factor protector debido a su importancia estadística en disminuir la mortalidad. Igualmente podemos observar que las variables que están relacionadas con el aporte de oxígeno al organismo $\left(\mathrm{PaO}_{2}\right.$ corregido, la relación $\mathrm{PaO}_{2} / \mathrm{PAO}_{2}$ y la $\mathrm{PAO}_{2}$ ), se destacan como condiciones de favorabilidad en el resultado final. Adicionalmente, lo anterior se evidencia en una relación sustancial con el FiO2, pues si la condición de normalidad está acompañada con requerimientos de $\mathrm{FiO}_{2}$ por debajo del 50\%, el resultado es favorable, pero si para la misma condición de normalidad es necesario $\mathrm{FiO}_{2}$ mayor al 50\%, el resultado final no es tan favorable. La interpretación anterior puede estar asociada con ausencia de compromiso de los órganos de la caja torácica por trauma y por ello, la suplencia de oxigeno no es mayor a $\mathrm{FiO}_{2}$ al 50\%. Se debe tener en cuenta que los pacientes más graves o con trauma de tórax, pueden tener registros de normalidad o inclusive supra normalidad (requerimiento de ventilación mecánica y presión positiva espiratoria final) en los valores relacionados con el aporte de oxigeno $\left(\mathrm{PaO}_{2}\right.$ corregido, la relación $\mathrm{PaO}_{2} / \mathrm{PAO}_{2}$ y la $\mathrm{PAO}_{2}$ ), pero debido a la gravedad del trauma, el resultado final suele ser desfavorable ${ }^{18,25}$.

La presión positiva espiratoria final aportada por la ventilación mecánica, permite garantizar una adecuada PAO2, pero no solo se debe tener en cuenta como parámetro aislado. Este oxígeno disponible en el alveolo debe cruzar la membrana alveolo-capilar, el oxígeno debe difundirse a través del alveolo y ser transportado hacia el organismo; a esto hace referencia la relación $\mathrm{PaO}_{2} / \mathrm{PAO}_{2}$. Esta variable está reportada en la tirilla de gases arteriales, y por ello debe ser tenido en cuenta a la hora de hacer la evaluación integral de los pacientes admitidos en la $\mathrm{UCl}$ con trauma, debido a su relación con la supervivencia. Si bien muchas veces se utilizan otras variables importantes como el lactato, para guiar la reanimación y el manejo de un paciente crítico, la invitación es para aprovechar el reporte de gases sanguíneos en forma integral y en especial, retomar el uso de la relación $\mathrm{PaO} 2 / \mathrm{PAO}_{2}{ }^{18,25}$. Finalmente el análisis de las variables como $\mathrm{PaO}_{2}, \mathrm{PAO}_{2}, \mathrm{PaO}_{2} \mathrm{PAO}_{2}$ y FC cuantificadas por gasometría, puede orientar el desenlace de su estado vital y pronóstico. Esta evaluación debe realizarse idealmente al ingreso del paciente a la institución.

\section{ConClusiones}

Este estudio logró demostrar mediante un análisis multivariado que la saturación de oxigeno y frecuencia cardiaca corresponden a variables predictivas de mortalidad, y las variables presión alveolar de $\mathrm{O}_{2}$ e índice arterio/alveolar de $\mathrm{O}_{2}$, son factores protectores de mortalidad, por lo que deben ser los blancos terapéuticos de corrección inmediata $^{23}$. Las escalas de severidad como APACHE II y el índice de shock, deben ser entendidos como escalas de medición del grado de intervención requerida por un paciente críticamente enfermo y no como predictores de mortalidad, puesto que su aplicación determina el grado y tipo de intervención requerido por cada paciente. Su ausencia puede determinar la probabilidad de muerte $\mathrm{e}^{26-28}$.

La invitación se convierte para los médicos intensivistas y de los servicios de trauma, que todo diagnóstico de trauma va de la mano con una evaluación del equilibrio ácido-base y una valoración integral del aporte de oxígeno a los tejidos para predecir tanto el desenlace como el pronóstico ${ }^{24}$.

\section{Consideraciones Éticas}

El estudio contó con los avale bioéticos del Comité de Bioética de la Universidad Tecnológica de Pereira y del Comité de Bioética del Hospital Universitario San Jorge.

\section{Financiamiento}

Los autores declaran que el presente estudio fue autofinanciado y no recibió apoyo económico de ninguna institución.

\section{CONFLicto de InTERÉS}

Los autores no declaran ningún conflicto de interés con los resultados del presente trabajo. 


\section{ReferenCias Biblográficas}

1. León HE. Aspectos epidemiológicos del trauma en Colombia. Arch med. 2004;4(9):55-62.

2. Instituto Nacional de Medicina Legal y Ciencias Forenses. Forensis Datos para la vida. Bogotá: Grupo Centro de Referencia Nacional sobre Violencia [actualizada en junio de 2012: consultada el 24 de Noviembre de 2012]. Disponible en: http:/ www.medicinalegal.gov.co/forensis .

3. Instituto Nacional de Medicina Legal y Ciencias Forenses. Forensis Datos para la vida. Bogotá: Grupo Centro de Referencia Nacional sobre Violencia [actualizada en julio de 2014: consultada el 25 de Mayo de 2015]. Disponible en: http://www. medicinalegal.gov.co/forensis .

4. Instituto Nacional de Medicina Legal y Ciencias Forenses Regional Occidente. Violencia y accidentalidad en Risaralda. Pereira: Grupo Centro de Referencia Regional sobre Violencia [actualizada en junio de 2011: consultada el 24 de Noviembre de 2012]. Disponible en: http://www.medicinalegal.gov.co/ violencia-y-accidentalidad-risaralda .

5. Shere-Wolfe RF, Galvagno SM, Grissom TE. Critical care considerations in the management of the trauma patien following initial resuscitation. Scand J Trauma Resusc Emerg Med. 2012;20:68.

6. Huang YC. Monitoring oxygen delivery in the critically Il. Chest. 2005;128(5 Suppl 2):554S-60

7. Gem Premier 3000 Manual.

8. Edwards SL. Pathophysiology of acid base balance: The theory practice relationship. Intensive Crit Care Nurs. 2008; 24(1):28-38.

9. Smith-Erichsen N, Kofstad J, Ingvaldsen B. Acid-base disturbances in intensive care patients. Tidsskr Nor Laegeforen. 2010; 130(15):1471-4.

10. Baylis C, Till C. Interpretation of arterial blood gases. Surg (Oxford). 2009;27(11):470-4.

11. Aristizábal-Salazar RE, Calvo-Torres LF, Valencia-Arango LA, Montoya-Cañón M, Barbosa-Gantiva O, Hincapié-Baena V. Equilibrio Ácido-Base: el mejor enfoque clínico. Rev Colomb Anestesiol. 2015;43:219-24.

12. Park M, Maciel AT, Noritomi DT, Pontes de Azevedo LC, Taniguchi LU, daCruz Neto LM. Effect of PaCo2 variation on standard base excess value in critically ill patients. J Crit Care 2009; 24(4):484-91.

13. Morgan TJ. Invited commentary: Putting standard base excess to the test. J Crit Care. 2009; 24(4):492-3.

14. Kofstad J. Base excess: a historical review-has the calculation of base excess been more standardized the last 20 years? Clin Chim Acta. 2001; 307(1-2):193-5.

15. Mentel A, Bach F, Schüler J, Herrmann W, Koster A, Crystal GJ, et al. Assessing Errors in the Determination of Base Excess. Anesth Analg. 2002; 94(5):1141-8.

16. Greenbaum J, Nirmalan M. Acid-base balance: The traditional approach. Curr Anesth Crit Care. 2005; 16(3):137-42.

17. Kratz A, Ferraro M, Sluss PM, Lewandrowski KB. Normal Reference Laboratory Values. N Engl J Med. 2004; 351(15):154863. 1. Karbing D, Kjaergaard SR, Smith B, Espersen K, Allerod $\mathrm{C}$, Andreassen S, Rees S. Variation in the $\mathrm{PaO} 2 / \mathrm{FiO} 2$ ratio with FiO2: mathematical and experimental description, and clinical relevance. Crit Care. 2007; 11(6):R118

18. Karbing D, Kjaergaard SR, Smith B, Espersen K, Allerod C, Andreassen S, Rees S. Variation in the $\mathrm{PaO} 2 / \mathrm{FiO} 2$ ratio with FiO2: mathematical and experimental description, and clinical relevance. Crit Care. 2007; 11(6):R118.

19. Berg MJ, Fynbo C, Christiansen S, Jensen R, Lemeshow S. Comparison of Charlson comorbidity index with sAPs and APACHE scores for prediction of mortality following intensive care. Clin Epidemiol. 2011; 3: 203-11.

20. Quach S, Hennessy DA, Faris P, Fong A, Quan H, Doig C. A comparison between the APACHE II and Charlson Index Score for predicting hospital mortality in critically ill patients. BMC Health Serv Res. 2009; 9:129.

21. Dossett LA, Redhage LA, Sawyer RG, May AK. Revisiting the Validity of APACHE II in the Trauma ICU: Improved Risk Stratification in Critically Injured. Injury. 2009; 40(9):993-8.

22. Knaus WA, Draper EA, Wagner DP, Zimmerman JE. APACHE II: a severity of disease classification system. Crit Care Med. 1985 13(10):818-29.

23. Chalya PL, Gilyoma JM, Dass RM, Mchembe MD, Matasha M, Mabula JB, et al. Trauma admissions to the intensive care unit at a reference hospital in Northwestern Tanzania. Scand J Trauma Resusc Emerg Med. 2011; 19:61.

24. Raum MR, Nijsten MW, Vogelzang M, Schuring F, Lefering R, Bouillon B, et al. Emergency trauma score: An instrument for early estimation of trauma severity. Crit Care Med. 2009; 37 (6): 1972-7.

25. Aristizábal RE, Martínez JW, Montoya M, Barbosa O, Calvo LF, Valencia LA, et al. Relación del aporte de oxígeno y la supervivencia del paciente con shock en UCI. Investigaciones Andinas. 2012;14 (25):588-600.

26. Vincent JL, Opal SM, Marshall JC. Ten reasons why we should NOT use severity scores as entry criteria for clinical trials or in our treatment decisions. Crit Care Med. 2010; 38(1):283-7.

27. Nguyen HB,Van Ginkel C,Batech M,Banta J, Corbett SW. Comparison of Predisposition, Insult/Infection, Response, Organ dysfunction, Acute Physiology And Chronic Health Evaluation II, and Mortality in Emergency Department Sepsis inpatients meeting criteria for early goal-directed therapy and the severe sepsis resuscitation bundle. J Crit Care. 2011; 27(4):362-9

28. Wenner JB, Norena M, Khan N, Palepu A, Ayas NT, Wong H, et al. Reliability of intensive care unit admitting and comorbid diagnoses, race, elements of Acute Physiology and Chronic Health Evaluation II score, and predicted probability of mortality in an electronic intensive care unit database. J Crit Care. 2009; 24(3)401-7. 